Proceedings of International Mathematical Sciences

ISSN:2717-6355, URL:HTTPS://DERGIPARK.ORG.TR/TR/PUB/PIMS

Volume II Issue 2 (2020), Pages 90-95. Doi: 10.47086/Pims.778004

\title{
ON THE STABILITY OF NONLOCAL BOUNDARY VALUE PROBLEM FOR SCHRÖDINGER-PARABOLIC EQUATIONS
}

\author{
YILDIRIM OZDEMIR* AND MUSTAFA ALP** \\ *DUZCE UNIVERSITY, DUZCE, TURKEY. ORCID NUMBER:0000-0003-2767-522X \\ **DUZCE UNIVERSITY, DUZCE, TURKEY. ORCID NUMBER:0000-0001-7299-4487
}

\begin{abstract}
In the present article, a problem for a Schrödinger-parabolic equation with nonlocal boundary condition is considered. The stability estimates are established for the solution of nonlocal boundary value problem for Schrödingerparabolic equation. The first and second order of accuracy difference schemes are used for approximate solutions of nonlocal boundary value problem. An example is considered and some error results of numerical experiments are presented in order to verify theoretical statements.
\end{abstract}

\section{INTRODUCTION}

In the present paper, the nonlocal boundary value problem (NBVP)

$$
\left\{\begin{array}{l}
\frac{d u(t)}{d t}+A u(t)=f(t)(0 \leq t \leq 1), \\
i \frac{d u(t)}{d t}+A u(t)=g(t)(-1 \leq t \leq 0), \\
u(-1)=\alpha u(\mu)+\varphi, 0<\mu \leq 1
\end{array}\right.
$$

for differential equations of Schrödinger-parabolic type in a Hilbert space $H$ with self-adjoint positive definite operator $A$ is considered.

It is well known that various NBVPs for the Schrödinger-parabolic equations can be reduced to problem 1.1 .

A function $u(t)$ is called a solution of the problem (1.1) if the following conditions are satisfied:

i. $u(t)$ is continuously differentiable on the segment $[-1,1]$. The derivative at the endpoints of the segment are understood as the appropriate unilateral derivatives.

ii. The element $u(t)$ belongs to $D(A)$ for all $t \in[-1,1]$, and the function $A u(t)$ is continuous on the segment $[-1,1]$.

iii. $u(t)$ satisfies the equations and nonlocal boundary condition (1.1).

2020 Mathematics Subject Classification. 2010 MSC: 65L10, 34B10, 65M12.

Key words and phrases. partial differential equation; nonlocal boundary value problem; stability.

(C)2020 Proceedings of International Mathematical Sciences.

Submitted on August 07th, 2020. Published on 12.30.2020. Communicated by Sahin UYAVER. 
In this study, the stability estimates for the solution of the problem (1.1) for the Schrödinger-parabolic equation are established.

Methods of solutions of NBVPs for PDEs and PDEs of mixed type have been studied extensively by many researches (see, e.g., [1-[12] and the references given therein).

\section{THE MAIN THEOREM ON STABILITY}

The goal of this section is to obtain the stability estimates for Schrödingerparabolic equations. In applications, the stability estimates of mixed type NBVP for Schrödinger-parabolic equations are constructed. On the other hand, all theoretical statements are supported by the results of numerical experiments.

Theorem 2.1. Let $\varphi \in D(A)$. Let $f(t)$ and $g(t)$ are continuously differentiable functions on $[0,1]$ and $[-1,0]$, respectively. Then, problem (1.1) has a unique solution and

$$
\begin{gathered}
\max _{-1 \leq t \leq 1}\|u(t)\|_{H} \leq M\left[\|\varphi\|_{H}+\max _{-1 \leq t \leq 0}\|g(t)\|_{H}+\max _{0 \leq t \leq 1}\|f(t)\|_{H}\right], \\
\max _{-1 \leq t \leq 1}\|A u(t)\|_{H} \leq M\left\{\|A \varphi\|_{H}+\|g(0)\|_{H}\right. \\
\left.+\max _{-1 \leq t \leq 0}\left\|g^{\prime}(t)\right\|_{H}+\|f(0)\|_{H}+\max _{0 \leq t \leq 1}\left\|f^{\prime}(t)\right\|_{H}\right\},
\end{gathered}
$$

inequalities hold. Here $M$ is independent of $f(t), t \in[0,1], g(t), t \in[-1,0]$ and $\varphi$.

Proof. First of all, we will obtain a formula for the solution of problem (1.1). It is very well known that there are unique solutions of the initial value problems

$$
\frac{d u(t)}{d t}+A u(t)=f(t)(0 \leq t \leq 1), u(0)=u_{0}
$$

and

$$
i \frac{d u(t)}{d t}+A u(t)=g(t)(-1 \leq t \leq 0), u(-1)=u_{-1}
$$

that is,

$$
u(t)=e^{-t A} u(0)+\int_{0}^{t} e^{-(t-s) A} f(s) d s, 0 \leq t \leq 1
$$

and

$$
u(t)=e^{i(t+1) A} u_{-1}-i \int_{-1}^{t} e^{i(t-s) A} g(s) d s,-1 \leq t \leq 0,
$$

respectively. Using formula (2.6), we get

$$
u(0)=e^{i A} u_{-1}-i \int_{-1}^{0} e^{-i s A} g(s) d s,-1 \leq t \leq 0 .
$$

After that we can write

$$
u(t)=e^{-t A}\left[e^{i A} u_{-1}-i \int_{-1}^{0} e^{-i s A} g(s) d s\right]+\int_{0}^{t} e^{-(t-s) A} f(s) d s, 0 \leq t \leq 1 .
$$

Now, using the nonlocal boundary condition

$$
u(-1)=\alpha u(\mu)+\varphi,
$$


we obtain the operator equation

$$
\begin{gathered}
\left\{I-\alpha e^{i A} e^{-\mu A}\right\} u_{-1} \\
=\left\{\alpha-i e^{-\mu A} \int_{-1}^{0} e^{-i s A} g(s) d s+\int_{0}^{\mu} e^{-(\mu-s) A} f(s) d s\right\}+\varphi .
\end{gathered}
$$

Here, the operator

$$
I-\alpha e^{i A} e^{-\mu A}
$$

has an inverse,

and

$$
T=\left(I-\alpha e^{i A} e^{-\mu A}\right)^{-1}
$$

$$
\|T\|_{H \rightarrow H} \leq M
$$

holds. The proof of this inequality is based on the following estimate

$$
\left\|-\alpha e^{-(\mu+i) A}\right\|_{H \rightarrow H}<1 .
$$

We have that

Then, it can be written that

$$
\left\|-\alpha e^{-(\mu+i) A}\right\|_{H \rightarrow H} \leq|-\alpha|\left|e^{-\mu \delta}\right|\left|e^{-i \delta}\right| \leq 1 .
$$

$$
\|T\|_{H \rightarrow H} \leq\left\|\left(I-\alpha e^{i A} e^{-\mu A}\right)^{-1}\right\|_{H \rightarrow H} \leq \frac{1}{1-|\alpha| e^{-\delta}} .
$$

Here, using the following definition

$$
\left(I-\alpha e^{i A} e^{-\mu A}\right)^{-1} u=\int_{\delta}^{\infty} \frac{1}{1-\alpha e^{i \lambda} e^{-\mu \lambda}} d E_{\lambda} u
$$

we get

$$
\begin{gathered}
\left\|\left(I-\alpha e^{i A} e^{-\mu A}\right)^{-1}\right\|_{H \rightarrow H} \leq \sup _{\delta \leq \lambda \leq \infty} \frac{1}{\left|1-\alpha e^{i \lambda} e^{-\mu \lambda}\right|} \\
\left|1-\alpha e^{i \lambda} e^{-\mu \lambda}\right| \geq 1-|\alpha|\left|e^{i \lambda}\right|\left|e^{-\mu \lambda}\right| \geq 1-|\alpha|\left|e^{-\mu \lambda}\right| \\
\geq 1-|\alpha|\left|e^{-\lambda}\right|=1-|\alpha| e^{-\delta} .
\end{gathered}
$$

Therefore,

$$
\left\|\left(I-\alpha e^{i A} e^{-\mu A}\right)^{-1}\right\| \leq \frac{1}{1-|\alpha| e^{-\delta}} \leq M .
$$

So, it has been proven the estimate (2.10).

Hence, we obtain the following formula from the operator equation 2.9

$$
u_{-1}=T\left(\alpha\left\{-i e^{-\mu A} \int_{-1}^{0} e^{-i s A} g(s) d s+\int_{0}^{\mu} e^{-(\mu-s) A} f(s) d s\right\}+\varphi\right) .
$$

Therefore, formulas 2.8, 2.6) and (2.11) are obtained for the solution of the problem (1.1). The proof of first part of the main theorem has been finished.

In the second part, proofs of estimates 2.1) and 2.2 will be given. Because of the symmetry properties of the operator $A$, we have the following estimates

$$
\left\|e^{ \pm i t A}\right\|_{H \rightarrow H} \leq 1, t \geq 0
$$


Firstly, the proof of the estimate 2.1) will be obtained. Using formula 2.11), we get

$$
\begin{gathered}
\left\|u_{-1}\right\|_{H} \leq\left\{\| ( I - \alpha e ^ { i A } e ^ { - \mu A } ) ^ { - 1 } \| _ { H \rightarrow H } \left(|i||\alpha|\left\|e^{-\mu A}\right\|_{H \rightarrow H} \int_{-1}^{0}\left\|e^{i A s}\right\|_{H \rightarrow H}\right.\right. \\
\left.\left.\times\|g(s)\|_{H} d s+|\alpha| \int_{0}^{\mu}\left\|e^{-(\mu-s) A}\right\|_{H \rightarrow H}\|f(s)\|_{H} d s+\|\varphi\|_{H}\right)\right\} \\
\leq M\left[\int_{-1}^{0}\|g(s)\|_{H} d s+\int_{0}^{\mu}\|f(s)\|_{H} d s+\|\varphi\|_{H}\right] .
\end{gathered}
$$

Hence,

$$
\left\|u_{-1}\right\|_{H} \leq M\left[\|\varphi\|_{H}+\max _{-1 \leq t \leq 0}\|g(t)\|_{H}+\max _{0 \leq t \leq 1}\|f(t)\|_{H}\right] .
$$

Using formula 2.8, we obtain

$$
\|u(t)\|_{H} \leq\left\|u_{-1}\right\|_{H}+\int_{-1}^{0}\|g(s)\|_{H} d s+\int_{0}^{t}\|f(s)\|_{H} d s .
$$

Hence,

$$
\|u(t)\|_{H} \leq M\left[\|\varphi\|_{H}+\max _{-1 \leq t \leq 0}\|g(t)\|_{H}+\max _{0 \leq t \leq 1}\|f(t)\|_{H}\right], 0 \leq t \leq 1 .
$$

Using formula 2.6, we get

$$
\|u(t)\|_{H} \leq\left\|u_{-1}\right\|_{H}+\int_{-1}^{t}\|g(s)\|_{H} d s,-1 \leq t \leq 0
$$

Hence,

$$
\|u(t)\|_{H} \leq M\left[\|\varphi\|_{H}+\max _{-1 \leq t \leq 0}\|g(t)\|_{H}+\max _{0 \leq t \leq 1}\|f(t)\|_{H}\right] .
$$

Therefore, using inequalities (2.14) and 2.15), we complete proof of inequality (2.1).

Secondly, the proof of the estimate 2.2 will be obtained. Using fomula (2.11) and integration by parts, we obtain

$$
\begin{aligned}
\left\|A u_{-1}\right\|_{H} \leq & M\left\{\|A \varphi\|_{H}+\|g(0)\|_{H}+\max _{-1 \leq t \leq 0}\left\|g^{\prime}(t)\right\|_{H}\right. \\
& \left.+\|f(0)\|_{H}+\max _{0 \leq t \leq 1}\left\|f^{\prime}(t)\right\|_{H}\right\}
\end{aligned}
$$

Now, we consider $-1 \leq t \leq 0$. Using formula 2.6) and integration by parts, we get

$$
\|A u(t)\|_{H} \leq\left\|A u_{-1}\right\|_{H}+\|g(0)\|_{H}+\max _{-1 \leq t \leq 0}\left\|g^{\prime}(t)\right\|_{H}
$$

Therefore, for $-1 \leq t \leq 0$ we obtain

$$
\begin{aligned}
\|A u(t)\|_{H} \leq & M\left[\|A \varphi\|_{H}+\|g(0)\|_{H}+\max _{-1 \leq t \leq 0}\left\|g^{\prime}(t)\right\|_{H}\right. \\
& \left.+\|f(0)\|_{H}+\max _{0 \leq t \leq 1}\left\|f^{\prime}(t)\right\|_{H}\right] .
\end{aligned}
$$

Finally, we consider $0 \leq t \leq 1$. Using formula 2.8 and integration by parts, we get

$$
\|A u(t)\|_{H} \leq M\left\{\|A \varphi\|_{H}+\|g(0)\|_{H}+\max _{-1 \leq t \leq 0}\left\|g^{\prime}(t)\right\|_{H}\right.
$$




$$
\left.+\|f(0)\|_{H}+\max _{0 \leq t \leq 1}\left\|f^{\prime}(t)\right\|_{H}\right\} .
$$

Using estimates (2.16), 2.17) and (2.18), we obtain (2.2). This completes the proof of the main theorem.

\section{NUMERICAL RESULTS AND ERROR ANALYSIS}

In this section, the nonlocal boundary value problem

$$
\left\{\begin{array}{l}
u_{t}-u_{x x}+u=(2-2 t) e^{-t^{2}} \sin x, 0<t<1,0<x<\pi, \\
i u_{t}-u_{x x}+u=(2-2 i t) e^{-t^{2}} \sin x,-1<t<0,0<x<\pi, \\
u\left(0^{+}, x\right)=u\left(0^{-}, x\right) ; u_{t}\left(0^{+}, x\right)=u_{t}\left(0^{-}, x\right), \\
u(-1, x)=u(1, x)+2 e^{-1} \sin x, 0 \leq x \leq \pi, \\
u(t, 0)=u(t, \pi)=0,-1 \leq t \leq 1
\end{array}\right.
$$

for a one dimensional Schrödinger-parabolic equation is considered. The first and second order of accuracy difference schemes are constructed for approximate solutions of nonlocal boundary value problem (3.1). We have second order difference equations with respect $n$ with matrix coefficients. For the computations modified Gauss elimination method [13] is applied.

The errors between exact and approximate solutions are computed by the formula

$$
E_{M}^{N}=\max _{1 \leq k \leq N-1}\left(\sum_{n=1}^{M-1}\left|u\left(t_{k}, x_{n}\right)-u_{n}^{k}\right|^{2} h\right)^{1 / 2} .
$$

Numerical solutions are recorded for different values of $N$ and $M$, where $u\left(t_{k}, x_{n}\right)$ represents the exact solution and $u_{n}^{k}$ represents the numerical solution at $\left(t_{k}, x_{n}\right)$. The results are shown in the Table 1 for $N=M=20,40,80$ and 160 .

\begin{tabular}{lcccc}
\hline Method & $N=M=20$ & $N=M=40$ & $N=M=80$ & $N=M=160$ \\
\hline FO DS & 0.0244 & 0.0133 & 0.0069 & 0.0035 \\
SO DS & 0.0060 & 0.0015 & $3.774 \times 10^{-4}$ & $9.4410 \times 10^{-5}$ \\
\hline
\end{tabular}

TABLE 1. Comparison of errors for the approximate solution of difference schemes

Hence, based on the numerical results of numerical experiments, one can conclude that the second order of accuracy difference schemes are more accurate than the first order of accuracy difference scheme.

Acknowledgments. The author would like to sincerely thank Prof. Dr. Hüseyin ÇAKALLI and Prof. Dr. Allaberen ASHYRALYEV for his valuable supports. 


\section{REFERENCES}

[1] A. Ashyralyev, and H. O. Fattorini, On uniform difference schemes for the second-order singular perturbation problem in Banach spaces, SIAM. J. Math. Anal., 23 (1992) 29-54.

[2] A. Ashyralyev, and N. Aggez, A note on the difference schemes of the nonlocal boundary value problems for hyperbolic equations, Numer. Func. Anal. Op., 25 (2004) 439-462.

[3] A. Ashyralyev, and Y. Ozdemir, Stability of difference schemes for hyperbolic-parabolic equations, Comput. Math. Appl., 50 (2005), 1443-1476.

[4] A. Ashyralyev, Nonlocal boundary-value problems or abstract parabolic equations: wellposedness in Bochner spaces, J. Evol. Equ., 6 (2006), 1-28.

[5] A. Ashyralyev, and Y. Ozdemir, On nonlocal boundary value problems hyperbolic-parabolic equations Taiwan. J. Math., 4 (2007), 1075-1089.

[6] A. Ashyralyev, and O. Gercek, Nonlocal boundary value problems of elliptic-parabolic differential and difference equations, Discrete. Dyn. Nat. Soc., 2008 (2008), 1-16.

[7] A. Ashyralyev, and A. Sirma, Nonlocal boundary value problems for Shrödinger equations, Comput. Math. Appl., 55 (2008), 392-407.

[8] A. Ashyralyev, and B. Hicdurmaz, A note on the fractional Schrödinger differential equatios, Kybernetes, 40 (2011), 736-750.

[9] A. Ashyralyev and O. Yıldırım, On multipoint nonlocal boundary value problems for hyperbolic differential and difference equations, Taiwan. J. Math., 14 (2010), 165-194.

[10] Y. Ozdemir, and M. Kucukunal, A note on nonlocal boundary value problems for hyperbolic Schrödinger equations, Abstr. Appl. Anal., 2012 (2012), 1-12.

[11] A. Ashyralyev, and O. Yıldırım, A note on the second order accuracy stable difference schemes for the nonlocal boundary value hyperbolic problem, Abstr. Appl. Anal., 2012 (2012), $1-29$.

[12] A. Ashyralyev, I. Karatay, and P. E. Soboloevskii, On well-posedness of the nonlocal boundary value problem for parabolic difference equations, Discrete. Dyn. Nat. Soc., 2 (2004), 273-286.

[13] A. A. Samarskii, and E. S. Nikolaev, Numerical Methods for Grid Equations, Vol. 2 Iterative Methods, Birkäuser, Basel, 1989.

YILDIRIM OZDEMIR,

Duzce University, Konuralp Campuss, Faculty of Arts and Sciences, Duzce, Turkey.

Phone: + (90) 544686 5722, OrCID NUMBer:0000-0003-2767-522X

Email address: yozdemir28@gmail.com

Mustafa Alp,

Duzce University, Duzce, Turkey. E-mail: mmustafa.alpp@gmail.com Orcid number:00000001-7299-4487

Email address: yozdemir28@gmail.com 